\title{
On-pump fibrillating heart mitral valve replacement with the SAPIEN ${ }^{\mathrm{TM}}$ XT transcatheter heart valve
}

\author{
Enrico Ferrari ${ }^{\mathrm{a}, *}$, Lars Niclauss ${ }^{\mathrm{a}}$, Didier Locca ${ }^{\mathrm{b}}$ and Carlo Marcucci ${ }^{\mathrm{c}}$
}

\author{
a Department of Cardiac Surgery, University Hospital of Lausanne, Lausanne, Switzerland \\ b Department of Cardiology, University Hospital of Lausanne, Lausanne, Switzerland \\ c Department of Cardiac Anaesthesia, University Hospital of Lausanne, Lausanne, Switzerland \\ * Corresponding author. Cardiac Surgery Unit, CHUV, Rue du Bugnon 46, CH-1011 Lausanne, Switzerland. Tel: +41-793101386; fax: +41-213142278; \\ e-mail: enricoferrari@bluewin.ch (E. Ferrari).
}

Received 30 April 2013; received in revised form 2 June 2013; accepted 11 June 2013

\begin{abstract}
In some high-risk patients, standard mitral valve replacement can represent a challenging procedure, requiring a risky extensive decalcification of the annulus. In particular, high-risk redo patients and patients with a previously implanted transcatheter aortic valve, who develop calcific mitral disease, would benefit from the development of new, minimally invasive, transcatheter or hybrid techniques for mitral valve replacement. In particular, mixing transcatheter valve therapies and well-established minimally invasive techniques for mitral replacement or repair can help in decreasing the surgical risk and the technical complexity. Thus, placing transcatheter, balloonexpandable Sapien ${ }^{\text {TM }}$ XT stent-valves in calcified, degenerated mitral valves through a right thoracotomy, a left atriotomy and on an on-pump fibrillating heart, represents an attractive alternative to standard surgery in redo patients, in patients with concomitant transcatheter aortic stent-valves in place and in patients with a high-risk profile. We describe this hybrid technique in detail.
\end{abstract}

Keywords: Transcatheter valve replacement • Mitral valve stenosis • Mitral valve replacement $•$ High-risk patients

\section{INTRODUCTION}

Mitral valve replacement with biological prosthesis is a valid treatment for elderly patients with non-repairable mitral disease [1-3]. However, mitral stenosis sometimes represents a challenging procedure, requiring valve replacement and extensive decalcification of the annulus, with risk of ventricular damage and bleeding [4] Thus, new, minimally invasive, transcatheter or hybrid techniques for mitral replacement could represent a further step in the treatment of selected high-risk patients.

Moreover, in recent years, high-risk patients with aortic stenosis have, worldwide, undergone the transcatheter implantation of aortic stent-valves and those developing a concomitant mitral disease will also take advantage of the development of new hybrid techniques [5]. The placement of the balloon-expandable Sapien $^{\text {TM }}$ XT (Edwards Lifesciences, Irvine, CA, USA) stent-valve in calcified mitral valves through a right thoracotomy, a left atriotomy and on an on-pump fibrillating heart represents an attractive new alternative to standard surgery in redo patients, patients with concomitant aortic stent-valves and patients with high-risk profile.

\section{PROCEDURAL DETAILS}

To explain the technique, an actual case is described as follows. A 60-year-old man with symptomatic mitral stenosis was screened for minimally invasive surgery at our institution. His clinical history showed rheumatic fever, previous mediastinal radiotherapy for lymphoma, chronic renal insufficiency and chronic obstructive pulmonary disease. Due to concomitant thorax irradiation and rheumatic fever, the patient also developed a severe symptomatic aortic stenosis: the screening, performed in 2010 in another European country, showed a 'porcelain' aorta and the presence of rare blood antibodies limiting transfusions in case of haemorrhage (GERBICH-positive). The standard aortic valve replacement was considered to be high-risk and the patient was successfully treated, in that centre, with a 26 mm CoreValve ${ }^{\circledR}$ (Medtronic, Minneapolis, MN, USA). After having considered the comorbidities and the limiting factors (porcelain aorta and antibodies) we identified in the on-pump fibrillating heart through a right thoracotomy, the best way to approach the mitral valve. However, the presence of the CoreValve $^{\circledR}$ and heavy mitral calcifications precluded some therapeutic options and the deployment of a balloonexpandable Sapien ${ }^{\mathrm{TM}}$ XT under direct view was considered the best option to reduce the cardiopulmonary bypass time, the risk of haemorrhage after extensive decalcification and the risk of CoreValve $^{\circledR}$ displacement during manipulations. The pre-operative transoesophageal echocardiogram (TEE) showed a calcified mitral valve with gradients of $29 \mathrm{mmHg}$ and $17 \mathrm{mmHg}$ (Fig. $1 \mathrm{~A}$ and B), a well-functioning CoreValve ${ }^{\circledR}$, pulmonary hypertension (mean pressure: $45 \mathrm{mmHg}$ ), a dilated left atrium and good left function. The coronary angiogram showed absence of coronary disease. The patient accepted a non-conventional approach and signed the consent. EuroSCOREs 1 (logistic) and 2 were 23 and 8\%, respectively. 


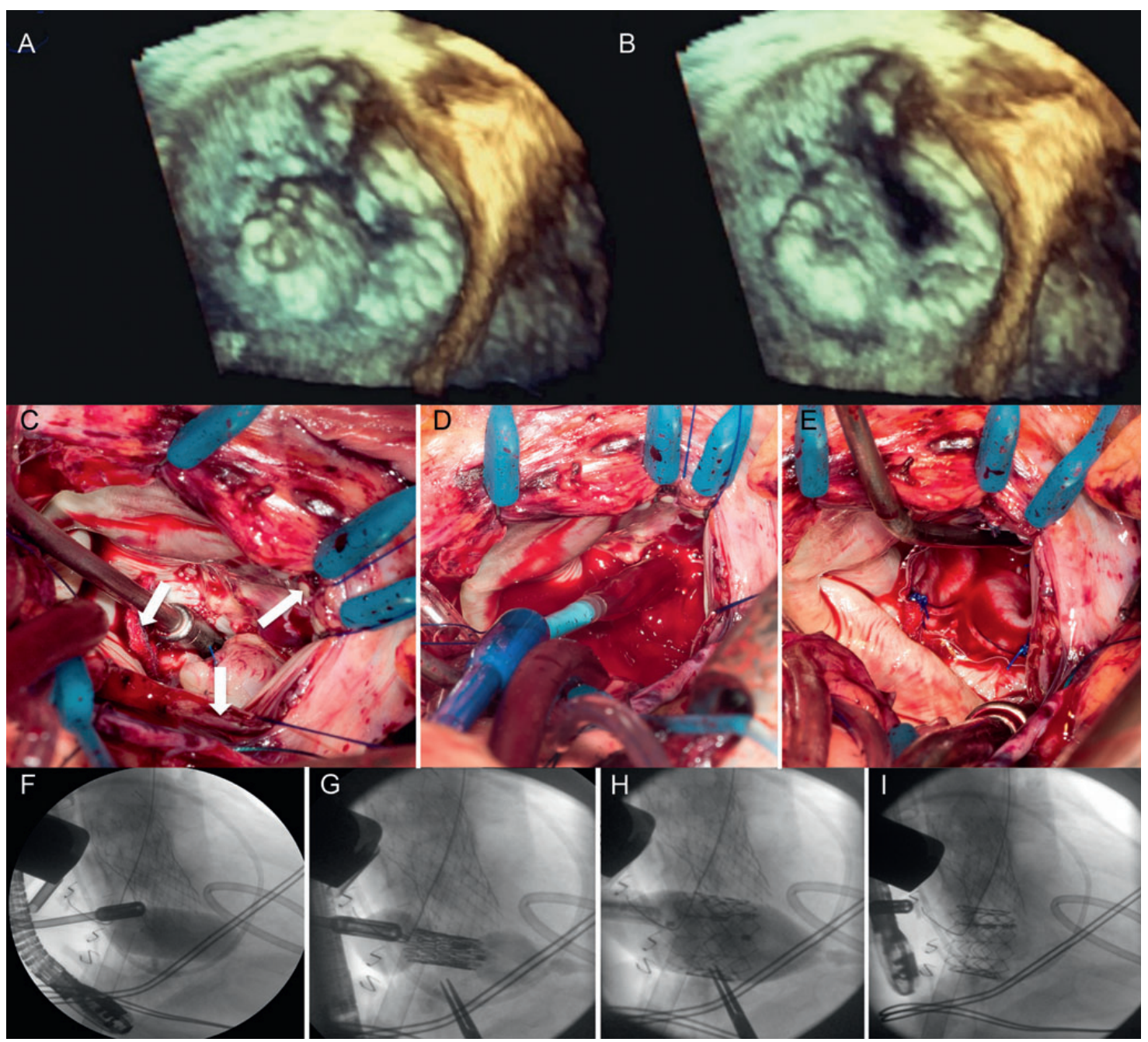

Figure 1: $(A, B)$ show a 3-dimensional echocardiogram of a stenotic mitral valve in systole and diastole. (C-E) are intra-operative views showing: (C) the calcified mitral valve with three 3-0 polypropylene U-stitches placed at the commissures and at the posterior leaflet midpoint (white arrows), (D) introduction of the delivery system with the crimped valve across the diseased mitral valve and $(\mathrm{E})$ the deployed stent valve in mitral position with the three stay sutures assuring the anchorage. F-I are fluoroscopic views showing: (F) mitral balloon valvuloplasty, (G) stent-valve placement and ballooning, (H) full inflation and (I) final result with the Sapien ${ }^{T M}$ XT in mitral position and the CoreValve ${ }^{\circledR}$ in aortic position.

The procedure was performed in the operating theatre with TEE and fluoroscopic monitoring: after the femoro-femoral cannulation and the right thoracotomy at fourth intercostal space, a left vent was placed in the right superior pulmonary vein and the fibrillator was switched on once on full bypass. The left atrium was opened $\left(\mathrm{CO}_{2}\right.$ use $)$ and the mitral valve exposed; this confirmed the diagnosis and the risk of performing extensive decalcifications. Following our strategy, we prepared a $29 \mathrm{~mm}$ Sapien $^{\text {TM }}$ XT valve while three 3-0 polypropylene $U$-stitches with pledgets were placed at commissures and at posterior leaflet midpoint to fix the deployed valve (Fig. 1C). Then, the mitral valvuloplasty was performed (Fig. 1F) and the Sapien ${ }^{\text {TM }}$ was placed across the mitral valve under direct and fluoroscopic view: half a centimetre of the bared stent was left in the atrium, where pre-placed U-stitches would be fixed, in order to prevent paravalvular leaks (Fig. 1D-G). After that, the Sapien ${ }^{\mathrm{TM}}$ was deployed (Fig. 1H) and fixed to the annulus using the three stitches (Fig. 1E-I). The left atrium was closed with the vent in place, the fibrillator was switched off and the cardiopulmonary bypass was weaned (fibrillating time: $20 \mathrm{~min}$; cardiopulmonary bypass time: $26 \mathrm{~min}$ ). Judging by our experience, the patient recovery was good, with rapid extubation and absence of paravalvular leak. The postoperative chest radiogram and echocardiogram revealed absence of interference between the two valves and an invasive aortic measurement showed a trans valvular peak gradient of less than 10 $\mathrm{mmHg}$ (Fig. 2).

\section{CONCLUSION}

Standard mitral replacement can be difficult in the presence of heavy calcifications and can lead to severe complications. Additionally, the concomitant presence of previously implanted transcatheter aortic valves (in particular the self-expandable stent-valves) can limit the access site, the decalcification and the placement of a standard prosthesis [1-5]. In this scenario, hybrid techniques may help in finding safer, faster and easier way to treat complex valve diseases. In particular, the use of the balloon-expandable Sapien ${ }^{\mathrm{TM}}$ valve in a calcified mitral valve appears easy, useful, and does not seem to interfere with the ventricular outflow tract and the left atrium because of its 

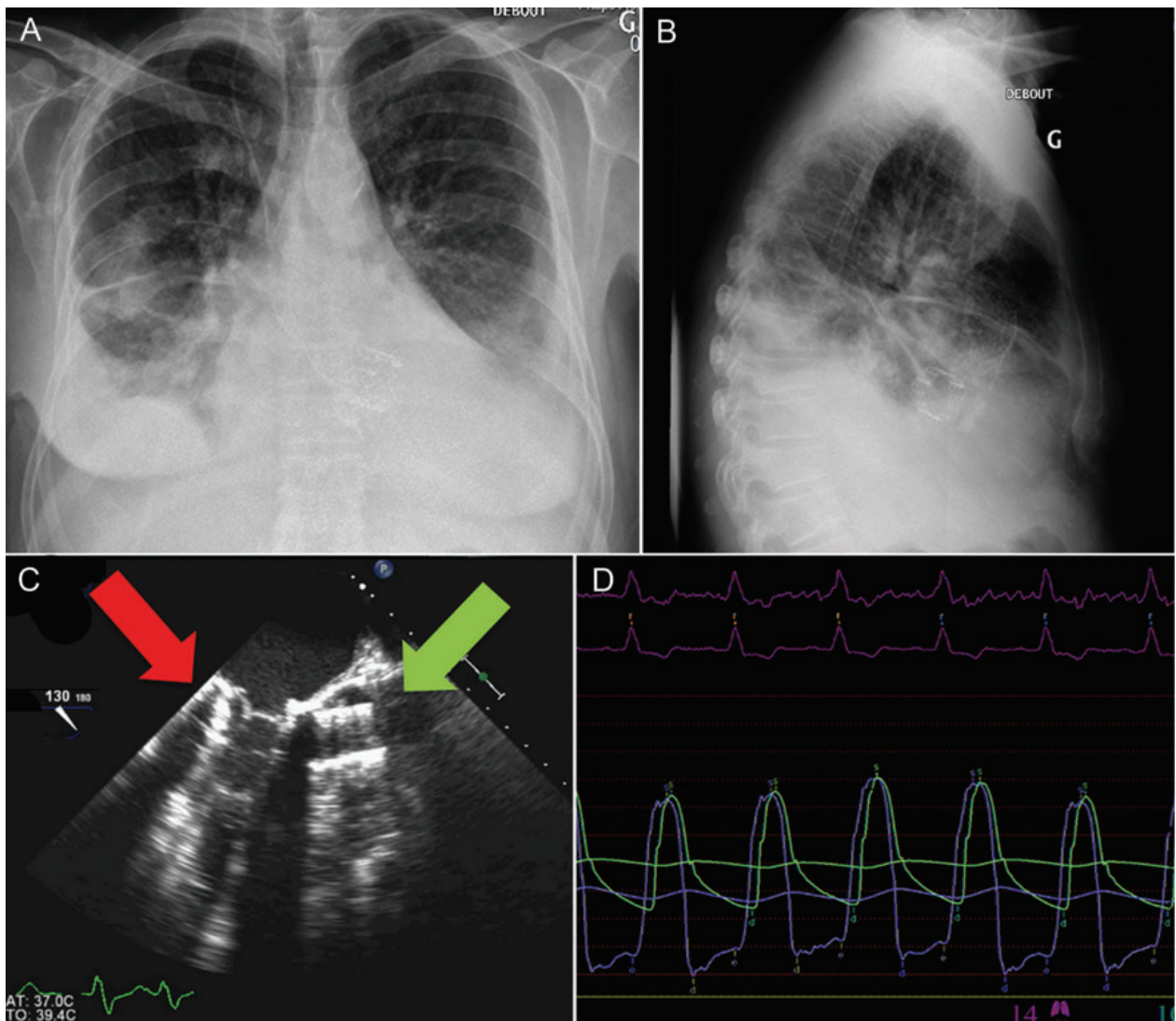

Figure 2: Postoperative imaging showing: (A and B) the thorax radiograms, (C) the transthoracic echocardiogram with the Sapien ${ }^{\mathrm{TM}}$ XT (red arrow) and the CoreValve ${ }^{\circledR}$ (green arrow) and (D) the invasive intracardiac left pressures showing a low trans-aortic gradient.

low-profile design. In our limited experience, the short delivery system was easy to manipulate through the thoracotomy, did not require the Ascendra ${ }^{\mathrm{TM}}$ sheat, and the Sapien ${ }^{\mathrm{TM}}$ was easily placed and deployed under direct and fluoroscopic view (to control interference with the stent-valve in the aortic position). We used three stitches to secure the valve at the annulus but it is likely that the Sapien ${ }^{\mathrm{TM}}$ can remain in place without surgical fixation (more laboratory tests are required before proceeding with transapical or direct transatrial off-pump procedures). This hybrid technique is useful in tricky and redo cases and the fact that the Sapien ${ }^{T M}$ could remain in place without direct fixation may pave the way for future transcatheter off-pump mitral replacements via a mini-thoracotomy or thoracoscopy, as already proposed for valve-in-valve procedures in cases of a degenerated bioprosthesis [6-8].

In conclusion, we should remember that rheumatic fever is still the main cause of mitral stenosis in several eastern, middleeastern and developing countries, and a hybrid on-pump fibrillating heart approach may represent an excellent alternative to standard surgery in high-risk patients.

\section{Conflict of interest: none declared.}

\section{REFERENCES}

[1] Jamieson WR, Gudas VM, Burr LH, Janusz MT, Fradet GJ, Ling H et al. Mitral valve disease: if the mitral valve is not reparable/failed repair, is bioprosthesis suitable for replacement? Eur J Cardiothorac Surg 2009;35:104-10.

[2] Mykén PS, Bech-Hansen O. A 20-year experience of 1712 patients with the Biocor porcine bioprosthesis. J Thorac Cardiovasc Surg 2009;137:76-81.

[3] Ayegnon KG, Aupart M, Bourguignon T, Mirza A, May MA, Marchand M. A 25-year experience with Carpentier-Edwards Perimount in the mitral position. Asian Cardiovasc Thorac Ann 2011;19:14-19.

[4] Kitamura T, Fukuda S, Sawada T, Miura S, Kigawa I, Miyairi T. Repeated mitral valve replacement in a patient with extensive annular calcification. J Cardiothorac Surg 2011;6:149.

[5] Flannery LD, Lowery RC Jr, Sun X, Satler L, Corso P, Pichard A et al. Transseptal mitral valve replacement after transcatheter aortic valve implantation. Tex Heart Inst J 2012;39:671-75.

[6] Cheung A, Webb JG, Barbanti M, Freeman M, Binder RK, Thompson C et al. 5-Year Experience With Transcatheter Transapical Mitral Valve-in-Valve Implantation for Bioprosthetic Valve Dysfunction. J Am Coll Cardiol 2013; 61:1759-66.

[7] Cheung A, Al-Lawati A. Transcatheter mitral valve-in-valve implantation: current experience and review of literature. Curr Opin Cardiol 2013;28:181-86.

[8] Schaefer U, Frerker C, Schewel D, Thielsen T, Meincke F, Kreidel F et al. Transfemoral and transseptal valve-in-valve implantation into a failing mitral xenograft with a balloon-expandable biological valve. Ann Thorac Surg 2012;94:2115-18. 